\title{
Influencia de las Narconovelas en el desarrollo de conductas desafiantes en adolescentes de secundaria de Estelí, Nicaragua
}

\author{
Anhyel Dayana Arróliga Flores ${ }^{1}$ \\ Ariely Gesarela Càlix Rivera² \\ María Fernanda Gómez Talavera ${ }^{3}$ \\ Franklin Solís Zúniga ${ }^{4}$
}

\section{RESUMEN}

La influencia que tienen las narconovelas en la población de adolescentes, es una temática poco abordada por los investigadores, a pesar de tener relevancia social que engloba distintas áreas del conocimiento, entre ellas, la Psicología. El presente documento, se enmarca en una investigación realizada con 28 adolescentes estudiantes de noveno y décimo grado del Instituto Nacional Francisco Luis Espinoza (INFLE) de Estelí, en el período 2016. El propósito fue determinar la influencia de las narconovelas en el desarrollo de conductas desafiantes en las y los adolescentes. El enfoque metodológico fue mixto con prevalencia cualitativa. Las técnicas utilizadas para la obtención de la información fueron: entrevistas, Cuestionario ESPERI, encuestas, socio-drama, guía de observación y técnica de listado libre. Los principales hallazgos revelan que las narconovelas con contenidos violentos predominantes, atraen la atención de los adolescentes influyendo así, en la adopción de conductas propias de los personajes. Así mismo, se evidencia que los adolescentes que ven este tipo de tele novelas durante períodos entre tres y cinco años, presentan conductas desafiantes. A partir de los resultados obtenidos en el estudio y a fin de incidir en el abordaje de esta temática con adolecentes, se elaboró un manual de intervención en conductas desafiantes en adolescentes dirigido a Psicólogos y Consejeros Escolares, como propuesta de trabajo para el centro educativo.

Palabras Claves: Narconovelas, Conductas desafiantes, Adolescentes, Influencia.

Recibido: 28 de marzo de 2017

Aceptado: 02 de junio de 2017

1 Egresada de la Licenciatura en Psicología. UNAN-Managua, FAREM-Estelí.

Correo electrónico: adar061194@yahoo.com

2 Egresada de la Licenciatura en Psicología. UNAN-Managua, FAREM- Estelí.

Correo electrónico: calixariely@yahoo.com

3 Egresada de la Licenciatura en Psicología UNAN-Managua, FAREM- Estelí.

Correo electrónico: gomezm861@yahoo.es

4 Docente UNAN-Managua, FAREM-Estelí. Correo electrónico: franksolis23@yahoo.com 


\title{
Influence of Narco soap operas on the development of challenging behaviors in adolescents
}

\begin{abstract}
The influence that narco soup operas have on the young population is a subject that has been scarcely studied, despite its social relevance that encompasses different areas of knowledge, for instance Psychology. This research was carried out with 28 ninth and tenth graders from the Francisco Luis Espinoza National Institute (INFLE) of Estelí, during 2016. The purpose was to determine the influence of narco soup operas in the development of teenagers behaviors. The methodological approach was mixed with qualitative prevalence. The techniques used to obtain the information were: interviews, ESPERI Questionnaire, surveys, socio-drama, observation guide and free listing technique. The main findings revealed that narco soup operas with predominant violent contents attract the attention of adolescents, thus influencing the adoption of behaviors characteristic of the actors. Likewise, it is evident that teenagers who watch this type of soup operas for periods between three and five years, present challenging behaviors. From the results obtained in the study and in order to influence the approach of this issue with adolescents, an intervention handdbook was made. This handbook aims to manage challenging behaviors in adolescents directed to Psychologists and School Counselors, as a proposal for the school.
\end{abstract}

Keywords: Narco Soap Operas, Challenging Behaviors, Adolescents, Influence. 


\section{INTRODUCCIÓN}

Las narconovelas empezaron a trasmitirse en Nicaragua desde el año 2008. La primera telenovela con este contenido, fue la llamada "Sin Tetas No Hay Paraíso", la cual rápidamente ganó popularidad entre la población nicaragüense y la comunidad latinoamericana.

La trama de este tipo de telenovelas, gira alrededor del narcotráfico (venta y consumo de estupefacientes) con altas dosis de violencia, trata de personas, abuso de poder, prostitución, corrupción y asesinatos. Estos temas son presentados de manera deliberada, sin restricción alguna de canales y horarios, por lo que, desde el niño más pequeño hasta el adulto puede visualizar este tipo de programación (Pérez, 2016, pág. 87).

En los hallazgos de la investigación Influencia de las narconovelas en el pensamiento y comportamiento de estudiantes adolescentes de dos colegios del Cantón Cuenca, la influencia de las narconovelas en las y los adolescentes, el autor lo resume en tres aspectos generales: modelo de vida capitalista, idea del cuerpo y las relaciones sociales efímeras; en donde las conductas de los adolescentes se ven modificadas de manera negativa a partir del modelo que las narconovelas ofrecen (Cárdenas Roldán, 2016, pág. 43).

Por otra parte, en la investigación Análisis de los discursos y contenidos de las narconovelas y el impacto que éstas han tenido en los adolescentes de 15 a 17 años que habitan en el sur de Quito, los resultados refieren que los adolescentes se han sentido de alguna manera identificados con uno o varios de los personajes de las narconovelas, deseando cambiar su apariencia física, siendo conscientes de los niveles de violencia manejado en este tipo de producciones. Se observó que las y los adolescentes implicados en el estudio, imitaban actitudes de personajes de las telenovelas. (Pérez, 2013)
Juárez, Salgado \& Salinas, (2009); realizaron la investigación denominada Influencia de las telenovelas en las actitudes de los y las adolescentes en Estelí, Nicaragua y los hallazgos determinan que en los y las adolescentes de nivel socioeconómico bajo, se da un proceso de identificación con determinados personajes de las telenovelas, que les incitan a cambios de actitudes en sus relaciones interpersonales. De igual manera, se encontraron actitudes de discriminación, segregación en grupo, y la reproducción de conductas negativas, formación en los barrios y aulas de clases de grupos de Divinas y Populares, en donde quienes vestían mejor, se consideraban más que los otros.

Desde la Psicología, es un deber el interesarse en estas realidades sociales y contribuir a desarrollar el sentido crítico de la población. El propósito de la investigación es determinar la Influencia de las narconovelas en el desarrollo de conductas desafiantes en las y los adolescentes del turno vespertino, Instituto Nacional Francisco Luis Espinoza (INFLE) de la ciudad de Estelí, en el período 2016.

Durante el proceso del estudio se elaboró un Manual de Intervención en Conductas Desafiantes en Adolescentes Dirigido a Consejeros y psicólogos escolares como propuesta al centro educativo en el que se desarrolló el estudio.

\section{MATERIALES Y MÉTODOS}

\section{Tipo de estudio}

La investigación se realizó a partir de un diseño mixto, combinando estrategias y técnicas cuantitativas y cualitativas. Para fines del estudio se seleccionaron estudiantes de noveno y décimo grado del Instituto Nacional Francisco Luis Espinoza (INFLE), Estelí, Nicaragua. 


\section{Población y Muestra}

La población en estudio, fueron estudiantes de secundaria del Instituto Nacional Francisco Luis Espinoza de Estelí. La muestra estuvo conformada por 28 adolescentes de noveno y décimo grado del Instituto. Se eligieron estos grupos, dado que cumplían la mayor parte de los criterios que se tenían previsto en el estudio. El proceso de selección de la muestra con estudiantes, se realizó mediante el muestreo no probabilístico, de tipo propositivo (Hernández, 2012, pág. 93) ya que se debía cumplir los siguientes criterios de selección:

- Ver series de narcotráfico.

- Presentar conductas desafiantes.

- Edades comprendidas entre los 14 y 18 años.

- Ser estudiante del Instituto Nacional Francisco Luis Espinoza Estelí (INFLE)

- Ser alumno activo de $3^{\circ}$ y $4^{\circ}$ año del Instituto (INFLE).

- Pertenecer al turno Vespertino.

- Disponibilidad para participar.

De igual manera se seleccionó un grupo de expertos del área de la Psicología de la ciudad de Estelí, tomando como muestra intencional a cinco Psicólogas y Psicólogos de la población total, que debían cumplir con los siguientes criterios de selección:

- Ser profesional de la ciencia de la conductaPsicología.

- Poseer conocimientos y experiencia de trabajo con adolescentes.

- Disponibilidad y voluntariedad para participar en el estudio.

\section{Métodos y técnicas para la recolección de datos}

Para la recolección de datos se aplicaron las técnicas de la encuesta, entrevista, cuestionario Esperi, técnica de listado libre, socio-drama y guía de observación del socio-drama. La entrevista constó de 18 ítems enfocados a conocer la percepción que tienen los adolescentes de las narconovelas. Para el caso de la encuesta ésta consta de cinco ítems dirigidos a conocer la población estudiantil que visualizan las narconovelas, así mismo, conocer el tiempo que dedican a estas programaciones.

El cuestionario Esperi constó de 48 ítems que valoran cuatro indicadores de conducta antisocial o desafiante en adolescentes (FundaciónO‘Belén, 2012). La entrevista a expertos constó de siete preguntas enfocadas a conocer el punto de vista de cada uno sobre esta temática. La guía de observación se compone de cuatro factores de evaluación de los adolescentes.

\section{Procesamiento y análisis de datos}

Una vez obtenidos los datos, estos fueron analizados según su naturaleza cuantitativa o cualitativa. Para la encuesta, se realizó un análisis cuantitativo de acuerdo a cada ítem, utilizando el software Microsoft Excel. En el caso de los resultados de las entrevistas, se realizó una trascripción fiel de la información obtenida, posteriormente se hizo una síntesis y análisis de los datos.

El cuestionario Esperi, se analizó de acuerdo a las normas evaluativas de validez y confidencialidad estipuladas por el test para su aplicación y revisión; posteriormente, fue ingresado a una base de datos construida en el paquete estadístico SPSS, donde se relacionaron estadísticamente las variables sexo, diagnóstico y tiempo dedicado a las narconovelas a través de la prueba estadística Chi Cuadrada.

La entrevista a expertos, fue procesada a través de una matriz de doble entrada donde se analizaron y compararon los principales resultados con la teoría. Al haber obtenido un resultado por cada entrevista se realizó un análisis de las propuestas sugeridas, la cual brindó pautas para la construcción del pequeño manual. En la técnica de listado libre se realizó un 
análisis cuantitativo y cualitativo, donde se valoraron las palabras asociadas al término narconovelas con los argumentos brindados por los informantes.

La guía de observación se analizó a través de una matriz de doble entrada, en donde se plasmó el análisis de lo observado durante el modelado en el socio-drama.

\section{RESULTADOS Y DISCUSIÓN}

\section{Percepción de las y los adolescentes del INFLE respecto a las narconovelas}

Los estudiantes, objetos de estudio, manifestaron que visualizan este tipo de programación en compañía de las familias, siendo estos principalmente los padres. Esto induce a pensar que los padres, que son los encargados de formar valores, principios morales y normas de comportamiento de los hijos e hijas, están siendo un modelo negativo, dado que no logran visualizar que estas novelas pueden tener consecuencias negativas en la conducta. Por otro lado, un porcentaje de los entrevistados, refiere ver narconovelas sin ninguna compañía, donde se constata la ausencia de los padres ante la regulación de lo que observan sus hijas e hijos a través de los medios de comunicación.

La mayoría coinciden en que las series de narcotráfico muestran escenas violentas en casi todas sus programaciones. Estas escenas están relacionadas a las acciones que realizan los narcotraficantes para conseguir sus objetivos, entre ellas: asesinatos a policías, trabajadores infieles, personas inocentes, maltrato hacia las mujeres, intimidación, abuso de poder. Por el contrario, una minoría de adolescentes que considera que las narconovelas no muestran ningún contenido o escena violenta.

Aunque la mayoría de los y las estudiantes hacen referencias negativas de las narconovelas, existe otro porcentaje que valoran las narconovelas como algo entretenido donde se viven escenas poco comunes en la vida de una persona particular, dejando a la población en suspenso, atrayendo así mayor audiencia. Así mismo, estos jóvenes perciben las narconovelas como algo positivo, viendo a los narcotraficantes como modelo a seguir ya que en la mayoría de las series los protagonistas se presentan con poder, fama, autoridad y dinero; influyendo así en la adopción de conductas negativas en la sociedad.

Es importante señalar que los hallazgos reflejan que los adolescentes del Instituto Nacional Francisco Luis Espinoza, reconocen aspectos negativos de este tipo de programación, los cuales han atraído su atención y de manera inconsciente están siendo influidos de tal manera que han incorporado conductas propias de los personajes en donde la percepción que tienen de ellas es producto de un esquema mental creado a partir de la temática y de igual manera de las experiencias vividas por los estudiantes.

En el gráfico que se muestra a continuación, se muestran la opinión sobre las palabras asociadas a las narconovelas, por las y los adolescentes.

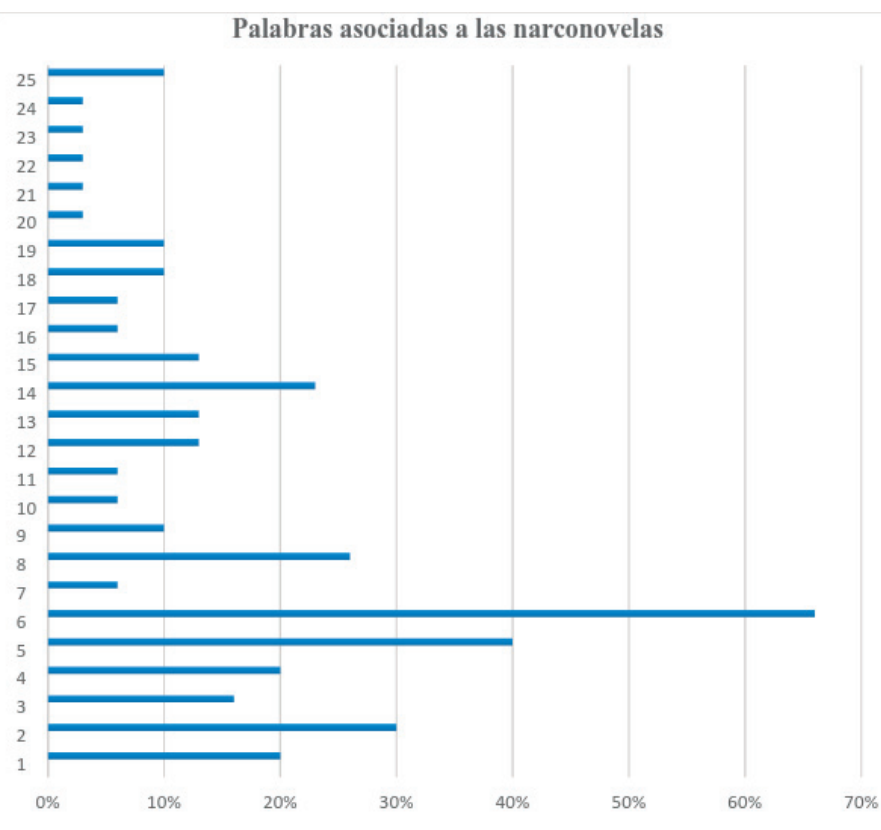

Grafico 1. Palabras Asociadas a las Narconovelas

Dentro de las palabras relacionadas con esta programación, mayormente se encuentra: drogas, con 
un $66 \%$, de la población, prostitución con un $40 \%$, un $26 \%$ asocia la palabra narcotráfico a las narconovelas $\mathrm{y}$ con un $30 \%$ se asocian las palabras sexo y la palabra muertecon un $23 \%$; así mismo una minoría de la población asocia las narconovelas a las palabras vulgaridad, miedo traición y guerra, con un 3\%.

Es importante resaltar que una minoría del $6 \%$ de la población, percibe como positivas las narconovelas al asociar las palabras entretenimiento y adrenalina, lo cual se puede interpretar como un factor predisponente a la observación masiva de narconovelas.

\section{Conductas desafiantes que presentan las y los estudiantes adolescentes del INFLE}

En el estudio se destacan algunas actitudes o comportamientos que manifiestan los adolescentes, que en su mayoría indica la presencia de conductas desafiantes; entre estas tenemos:

- Consumo de sustancias psicotrópicas como alcohol y marihuana.

- Venta de drogas o cosas robadas

- Huir de casa de sus padres

- Enfrentar problemas legales por distintos delitos.

- Robos en propiedad privada

- Irresponsabilidad

- Robos de amenaza con armas

- Destrucción de lo ajeno

- Inicio de incendios a propósito

- Actuar sin pensar en las consecuencias

- Distracción fácilmente

- Desobediencia

- Irrespeto de normas sociales y a personas mayores.

- Llevar la contraria

- Peleas constantes

- Humillar a otros

- Pertenencia a pandillas

- Mentiras constantes para evitar la ley

- Egoísmo

- Irritabilidad

- Crueldad con personas y animales sin inmutarse.
- Inestabilidad emocional y social

- Ansiedad.

La siguiente tabla hace referencia a la variable conducta desafiante relacionada con el nivel de diagnóstico que presentan los adolescentes, en la cual se utiliza la prueba estadística Chi-Cuadrada, obteniendo los siguientes resultados:

\section{Pruebas de chi-cuadrado de las variables conducta desafiante y nivel de Diagnostico}

\begin{tabular}{llll}
\hline & Valor & Gl & $\begin{array}{c}\text { Sig. asintótica } \\
\text { (bilateral) }\end{array}$ \\
\hline $\begin{array}{l}\text { Chi-cuadrado de } \\
\text { Pearson }\end{array}$ & $34,591^{\mathrm{a}}$ & 12 &, 001 \\
$\begin{array}{l}\text { Razón de } \\
\text { verosimilitudes }\end{array}$ & 21,320 & 12 &, 046 \\
$\begin{array}{l}\text { Asociación lineal por } \\
\text { lineal }\end{array}$ & 6,208 & 1 &, 013 \\
$\mathrm{~N}$ de casos válidos & 28 & & \\
\hline
\end{tabular}

Tabla 1. Prueba chi cuadrada conductas desafiantes y nivel de diagnóstico

Dados los resultados de la Chi-cuadrada respecto a las variables nivel de diagnóstico y conductas desafiantes se puede decir que existe una significancia estadística entre las variables, debido que el valor obtenido en la Chi Cuadrada es 34,591 que es mayor a 3.84; siendo significativo al nivel de 0.05 . Lo que indica que el nivel de diagnóstico es influido por el tipo de conducta desafiante que los adolescentes presentan.

La tabla de contingencia que se muestra a continuación, describe los niveles de diagnóstico por cada una de las conductas desafiantes, en donde se describe si existe o no una relación estadística entre ellas. 
Tabla de contingencia Conductas desafiantes * Nivel del diagnóstico

\begin{tabular}{|c|c|c|c|c|c|c|c|}
\hline & & & \multicolumn{4}{|c|}{ ¿Nivel del Diagnóstico? } & \multirow[t]{2}{*}{ Total } \\
\hline & & & Normal & Leve & Moderado & Severo & \\
\hline \multirow[t]{10}{*}{ Conductas desafiantes } & Disocial & Recuento & 0 & 1 & 5 & 6 & 12 \\
\hline & & $\begin{array}{l}\% \text { dentro de conductas } \\
\text { desafiantes }\end{array}$ &, $0 \%$ & $8,3 \%$ & $41,7 \%$ & $50,0 \%$ & $100,0 \%$ \\
\hline & Inatención e & Recuento & 0 & 4 & 5 & 2 & 11 \\
\hline & impulsividad & $\begin{array}{l}\% \text { dentro de conductas } \\
\text { desafiantes }\end{array}$ &, $0 \%$ & $36,4 \%$ & $45,5 \%$ & $18,2 \%$ & $100,0 \%$ \\
\hline & Pre-disocial & Recuento & 0 & 0 & 1 & 1 & 2 \\
\hline & & $\begin{array}{l}\% \text { dentro de conductas } \\
\text { desafiantes }\end{array}$ &, $0 \%$ &, $0 \%$ & $50,0 \%$ & $50,0 \%$ & $100,0 \%$ \\
\hline & Hiperactividad & Recuento & 0 & 0 & 0 & 1 & 1 \\
\hline & & $\begin{array}{l}\% \text { dentro de conductas } \\
\text { desafiantes }\end{array}$ &, $0 \%$ &, $0 \%$ &, $0 \%$ & $100,0 \%$ & $100,0 \%$ \\
\hline & Ninguno & Recuento & 2 & 0 & 0 & 0 & 2 \\
\hline & & $\begin{array}{l}\% \text { dentro de conductas } \\
\text { desafiantes }\end{array}$ & $100,0 \%$ &, $0 \%$ &, $0 \%$ &, $0 \%$ & $100,0 \%$ \\
\hline \multirow[t]{2}{*}{ Total } & & Recuento & 2 & 5 & 11 & 10 & 28 \\
\hline & & $\begin{array}{l}\% \text { dentro de conductas } \\
\text { desafiantes }\end{array}$ & $7,1 \%$ & $17,9 \%$ & $39,3 \%$ & $35,7 \%$ & $100,0 \%$ \\
\hline
\end{tabular}

Tabla 2. Tabla de contingencia de las variables conductas desafiantes y nivel de diagnóstico

En esta tabla está reflejado que los altos niveles de conductas desafiantes están remarcados en el trastorno Disocial con un 50\% correspondiente a 6 adolescentes respectivamente, perteneciente a la población con un Diagnóstico severo; el trastorno de inatención e Impulsividad posee un $45,5 \%$ correspondiente a 5 adolescentes con un Diagnóstico Moderado al igual que la conducta Disocial con un $41.7 \%$ equivalente a 5 estudiantes en total, indicando que del 100\% de la población un 39.3\% corresponde al nivel Moderado de estas conductas; y con un $36,4 \%$ que corresponde a 4 adolescentes, se encuentra el rango leve de la conducta de Inatención e impulsividad; Con un mínimo de 7.1\% equivalente a 2 personas sin ningún tipo de diagnóstico.

Por tanto, se considera que los adolescentes del INFLE de la ciudad de Estelí, presentan conductas desafiantes a partir de la observación de narconovelas, lo cual constata la teoría de Albert Bandura sobre el aprendizaje social mediante la observación. Esta teoría plantea que el adolescente presta atención a los refuerzos que genera una conducta, ya sea propia o de un modelo, posteriormente la reproduce, y al recibir refuerzo de esta, la incorpora como aprendizaje.

conductas desafiantes
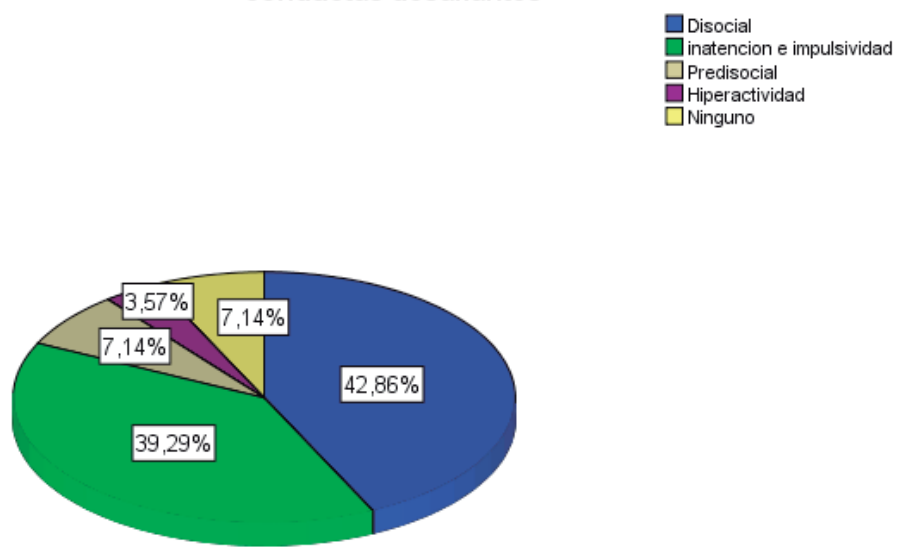

Gráfico 2. Conductas desafiantes que presentan los adolescentes 
El gráfico muestra que en relación a las conductas desafiantes que presentan la población en estudio, la mayoría presenta una conducta disocial y seguidamente se encuentra la conducta de inatención e impulsividad.

\section{Relación entre el tiempo dedicado a las narconovelas y el desarrollo de conductas desafiantes en las y los adolescentes}

En relación a la relación existente entre el tiempo dedicado a las narconovelas y el desarrollo de conductas desafiantes en las y los adolescentes, se describen a continuación las variables tiempo y desarrollo de conductas desafiantes.

Tabla de contingencia Tiempo dedicado a narconovelas * Conductas desafiantes

\begin{tabular}{|c|c|c|c|c|c|c|c|c|}
\hline & & & \multicolumn{5}{|c|}{ Conductas desafiantes } & \multirow[t]{2}{*}{ Total } \\
\hline & & & Disocial & $\begin{array}{l}\text { inatención e } \\
\text { impulsividad }\end{array}$ & Predisocial & Hiperactividad & Ninguno & \\
\hline \multirow{10}{*}{$\begin{array}{l}\text { ¿Hace } \\
\text { cuánto ve } \\
\text { narconovelas? }\end{array}$} & 1 año & Recuento & 0 & 1 & 0 & 0 & 2 & 3 \\
\hline & & $\begin{array}{l}\% \text { dentro de } \\
\text { conductas } \\
\text { desafiantes }\end{array}$ &, $0 \%$ & $9,1 \%$ &, $0 \%$ &, $0 \%$ & $100,0 \%$ & $10,7 \%$ \\
\hline & 2 & Recuento & 0 & 4 & 0 & 0 & 0 & 4 \\
\hline & años & $\begin{array}{l}\% \text { dentro de } \\
\text { conductas } \\
\text { desafiantes }\end{array}$ &, $0 \%$ & $36,4 \%$ &, $0 \%$ &, $0 \%$ &, $0 \%$ & $14,3 \%$ \\
\hline & 3 & Recuento & 2 & 4 & 1 & 1 & 0 & 8 \\
\hline & años & $\begin{array}{l}\% \text { dentro de } \\
\text { conductas } \\
\text { desafiantes }\end{array}$ & $16,7 \%$ & $36,4 \%$ & $50,0 \%$ & $100,0 \%$ &, $0 \%$ & $28,6 \%$ \\
\hline & 4 & Recuento & 6 & 0 & 0 & 0 & 0 & 6 \\
\hline & años & $\begin{array}{l}\% \text { dentro de } \\
\text { conductas } \\
\text { desafiantes }\end{array}$ & $50,0 \%$ &, $0 \%$ &, $0 \%$ &, $0 \%$ &, $0 \%$ & $21,4 \%$ \\
\hline & mas & Recuento & 4 & 2 & 1 & 0 & 0 & 7 \\
\hline & $\begin{array}{l}\text { de } 5 \\
\text { años }\end{array}$ & $\begin{array}{l}\% \text { dentro de } \\
\text { conductas } \\
\text { desafiantes }\end{array}$ & $33,3 \%$ & $18,2 \%$ & $50,0 \%$ &, $0 \%$ &, $0 \%$ & $25,0 \%$ \\
\hline \multirow[t]{2}{*}{ Total } & & Recuento & 12 & 11 & 2 & 1 & 2 & 28 \\
\hline & & $\begin{array}{l}\% \text { dentro de } \\
\text { conductas } \\
\text { desafiantes }\end{array}$ & $100,0 \%$ & $100,0 \%$ & $100,0 \%$ & $100,0 \%$ & $100,0 \%$ & $100,0 \%$ \\
\hline
\end{tabular}

Tabla 3. Tabla de contingencia: Variables Tiempo Dedicado a las Narconovelas y Conductas Desafiantes. 
La presente tabla de contingencia representa las variables, tiempo dedicado a las narconovelas $\mathrm{y}$ conductas desafiantes, con el fin de comprobar si el tiempo dedicado a este tipo de programación influye en el desarrollo de conductas desafiantes, encontrándose que: Dentro del grupo de adolescentes que lleva 1 año visualizando narconovelas un 9.1\% (1 persona) presenta conductas de inatención e impulsividad, reflejando que de las personas que no presentan ningún tipo de conducta desafiante se encuentra en este rango de tiempo dedicado a las narconovelas.

De igual manera, de las personas que han dedicado cuatro años a las narconovelas equivale a un $21.4 \%$ de la población total, en donde un $50 \%$ que corresponde a 6 personas presenta conducta Disocial, A su vez, de las personas que han dedicado más de 5 años a las narconovelas, corresponde un $25 \%$ de la población total; siendo que de la población total que presenta conducta Pre disocial, un 50\% (1persona), se encuentra en este lapso de tiempo, un $33.3 \%$ de la población que presenta conducta disocial y un mínimo de $18.2 \%$ de la población presenta intención e impulsividad.

Por lo que se puede resumir, que el mayor número de conductas desafiantes se da entre el rango de tres, cuatro y más de cinco años dedicados a las narconovelas. De esta manera se relaciona el tiempo dedicado a las narconovelas con el desarrollo de conductas desafiantes.

Prueba Chi cuadrada de las variables tiempo dedicado a las narconovelas y conductas desafiantes

Valor gl Sig. asintótica (bilateral)

\begin{tabular}{llll}
\hline Chi-cuadrado de Pearson & $35,992^{\mathrm{a}}$ & 16 &, 003 \\
Razón de verosimilitudes & 32,060 & 16 &, 010 \\
Asociación lineal por & 8,508 & 1 &, 004 \\
lineal & & & \\
N de casos válidos & 28 & & \\
\hline
\end{tabular}

a. 25 casillas (100.0\%) tienen una frecuencia esperada inferior a 5. La frecuencia mínima esperada es .11.

Tabla 4. Prueba de Chi cuadrada de las variables Tiempon dedicado a las narconovelas y Conductas desafiantes.
La tabla anterior nos muestra los resultados de la prueba estadística chi-cuadrada de acuerdo a las variables tiempo dedicado a las narconovelas y conductas desafiantes lo cual hace referencia que existe una significancia estadística entre las variables debido a que el valor expuesto es de 35.992 siendo este valor mayor a 3.84; el cual es significativo al nivel de 0.05 . Lo cual indica que el tiempo dedicado a las narconovelas influye en las conductas desafiantes manifestadas por las y los adolescentes.

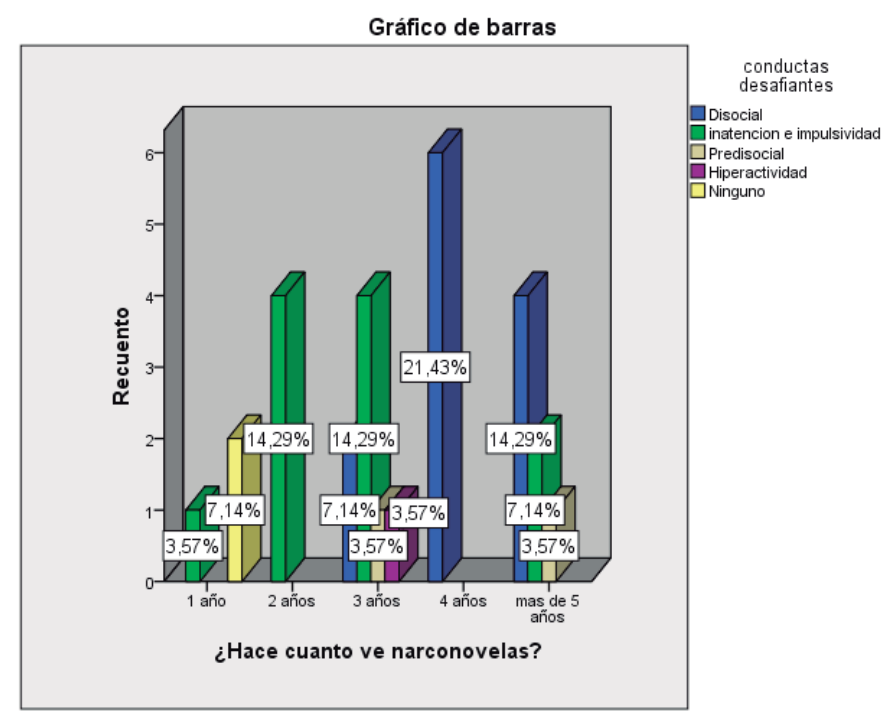

El gráfico anterior, refleja las variables tiempo dedicado a las narconovelas y conductas desafiantes, en donde se refleja que la conducta disocial, la cual es la más severa dentro de este tipo de conductas se encuentra entre cuatro y más de cinco años de tiempo dedicados a las narconovelas.

La conducta de inatención e impulsividad se encuentra mayor en las personas que han dedicado entre dos y tres años a este tipo de programación. Es importante destacar que de las personas que no presentan ningún tipo de conducta desafiante le han dedicado un año a estas novelas. Por tanto, se asume que las mayores conductas desafiantes se presentan en adolescentes que dedican mayor tiempo a ver este tipo de telenovelas. De esta manera, se puede decir que el tiempo si influye en el desarrollo de conductas desafiantes. 
Así mismo, se observó que los adolescentes que han dedicado entre tres y cinco años a la observación de narconovelas, presentan conductas desafiantes, las cuales han sido reforzadas entre coetáneos ya que parte de la aceptación dentro del grupo, es decir, se ha tomado como un moda común entre los adolescentes que va desde su forma de vestir hasta el maquillaje utilizado.

A través del análisis, se han constatado las teorías de Albert Bandura y Erick Erickson, si el individuo tiene un yo poco estructurado tiende a manifestar conductas que ha adquirido a partir del tiempo dedicado a la observación de narconovelas, propio del aprendizaje observacional; por ende es considerable que el tiempo dedicado influye en el desarrollo de conductas desafiantes.

\section{Intervención psicológica para el abordaje de conductas desafiantes en las y los adolescentes}

A partir de los datos recolectados en el proceso investigativo, y las sugerencias de algunos de los expertos, se construyó un manual llamado "Manual de intervención en conductas desafiantes en adolescentes dirigido a consejeros y Psicólogos escolares." El cual está basado en el enfoque conductista, en donde se plantean algunas técnicas para trabajar con los adolescentes de manera grupal en el aula de clase, abordando de igual manera a los padres de familia como principales responsables de la educación de sus hijos.

Dicho manual es producto del compromiso asumido al iniciar el proceso investigativo, el cual fue entregado a la directora del Instituto Nacional Francisco Luis Espinoza-INFLE-Estelí; para su utilización con los adolescentes del centro educativo.

\section{CONCLUSIONES}

El principal hallazgo fue que la percepción que tienen los adolescentes sobre las narconovelas se encuentra dirigida a aspectos superficiales sobre el contenido que se presenta; lo cual no les permite realizar un razonamiento lógico de lo que ocultan las novelas con contenido de narcotráfico, debido a la falta de sentido crítico de lo que los medios de comunicación presentan. Es decir, que se dejan llevar por aspectos que para ellos resultan atractivos, la fama, el dinero, belleza, lujos y el poder que conlleva este negocio, tomándolo como un modelo a seguir, creándose un interés por asumir este rol aunque el pago de ello sea perjudicar a otras personas y así mismos.

Los adolescentes consideran de su agrado este tipo de contenido, lo que les motiva a ver con mayor frecuencia las narconovelas, admirando a determinados personajes y adoptando en su personalidad modelos de comportamiento que no son aceptados socialmente dentro del contexto en que se desarrollan los estudiantes.

Los y las adolescentes presentan conductas desafiantes de inatención e impulsividad y disocial; por lo que se considera que la observación de narconovelas influye en el desarrollo de conductas desafiantes, tales como el consumo y venta de sustancias, robos, pertenencia a grupos juveniles, uso de vocabulario inadecuado, irrespeto de las normas sociales, irrespeto a las autoridades.

De igual manera, los adolescentes que han dedicado entre tres y cinco años a la observación de conductas desafiantes, han desarrollado conductas Disociales. Esto puede llevar a que estos adolescentes tengan un grado alto de riesgo de delinquir. En cambio los estudiantes que han dedicado menor tiempo a las narconovelas presentan otros tipos de conductas de menor riesgo, así como otros que no presentan conductas desafiantes. 


\section{AGRADECIMIENTOS}

A la Lic. María del Socorro Torres Olivas Delegada Municipal del Ministerio de Educación Estelí por su apoyo institucional mediante la autorización para llevar a cabo nuestro proceso investigativo en uno de los centros de educación secundaria.

A la Lic. Onelia Rivera Directora del Instituto Nacional Francisco Luis Espinoza por facilitarnos un espacio ameno para la realización del proceso investigativo; así mismo a los estudiantes de noveno y décimo grado F por su participación activa y voluntad en la etapa de fase de campo de nuestro estudio.

Agradecemos especialmente a nuestro maestro y amigo Ms.c. Franklin Solís Zúniga Tutor de nuestro estudio investigativo, quien nos brindó su preciado tiempo y apoyo incondicional en la realización de este trabajo, el cual con gran sabiduría, dedicación y esmero ha sabido guiarnos durante este proceso por su profesionalismo, humanismo, entrega y preocupación demostrada en nuestro crecimiento profesional, motivándonos a seguir adelante cada día a pesar de las dificultades presentadas en este periodo.

\section{REFERENCIAS BIBLIOGRAFICAS}

Cárdenas, Roldán. C.E. (2016). Influencia de las narconovelas en el pensamiento y comportamiento de estudiantes adolescentes de dos colegios del cantón Cuenca. Tesis para obtencion de titulo en Licenciatura de Socioologia., Universidad de Cuenca., Cuenca Ecuador.

Fundación O Belén. Cuestionario para la detección de los trastornos del comportamiento en niños y adolescentes “ESPERI”, Madrid. Universidad del Bosque.

Hernandez, Sampieri, R.; Fernández-Collado, C.; Baptista, Lucio, P. (2012). Metodologia de la investigacion. (4ta Ed.) Mc Graw Hill. México.

Juárez, Salgado, \& Salinas. (2009). Influencia de las telenovelas en las actitudes de los y las adolescentes. Esteli, Nicaragua.

Pérez, Villacis, L.A. (2013). Análisis de los discursos y contenidos de las narconovelas y el impacto que éstas han tenido en los niños de 15 a 17 años que habitan en el sur de Quito. Tesis licenciatura en Comunicación Social. Recuperado de http:// dspace.ups.edu.ec/bitstream/123456789/6511/1/ UPS-QTO5122.pdf 